\title{
Evaluation de la valeur alimentaire d'une variété de maïs waxy ensilé en plante entière et à destination des vaches laitières. Comparaison à 2 témoins : l'un isogène et l'autre dis gène
}

\author{
$\checkmark$ Legarto \\ Institut de l'Elevage, domaine d'Ognoas, 40190 Arthez d'Armagnac, France
}

La variabilité génétique du maïs est importante et étudiée de longue date. Parmi les mutants connus, citons les maïs cireux ou à gène waxy récessif. Ces maïs waxy ne contiennent que de l'amylopectine dans l'amidon du grain, alors que les grains normaux n'en possèdent que 70 à $75 \%$, le reste étant de l'amylose.

Nous avons voulu évaluer ce mutant par rapport à son témoin isogène (non mutant), du point de vue de sa production de biomasse et de sa valeur alimentaire pour vaches laitières.

Les mesures de productivité fourragère ont été réalisées sur 3 ans (1990 à 1992) en grandes et petites parcelles, tant en station experimentale qu'en ferme. Les estimations de valeurs alimentaires ont été effectuées dans 2 essais zootechniques en station expérimentale. Le premier essai a comparé un maïs waxy à son isogène (témoin) et le deuxième essai a reconduit ces deux modalités en y ajoutant un deuxième témoin génétiquement distants de la variété précédente (témoin dis gène).

Au champ, à même date de semis, la production de matière sèche de la plante entière est peu modifiée par le caractère waxy, alors que la production de grain est diminuée de 5 à $10 \%$ à cause d'une humidité accrue.

L'ensilabilité du maïs waxy reste bonne et équivalente à celle des témoins. Distribué à des vaches laitières, l'ensilage de maïs waxy a été aussi bien ingéré que celui des maïs témoins. Il a eu tendance à accroître la quantité de lait produit sans entraîner de dilution des matières utiles $(+1,5 \mathrm{~kg}$ de lait à $7 \% /$ vache / jour). L'augmentation du taux de matière grasse (TB) semble plus constante et répétable que celle du taux protéique (TP) (1 à $1,5 \mathrm{~g} / \mathrm{kg}$ de TB contre 0,4 à $0,8 \mathrm{~g} / \mathrm{kg}$ de TP). Le rapport TB/TP des laits produits n'est donc pas amélioré par l'effet waxy. Les mesures des profils fermentaires des jus de rumen ont permis en partie d'expliquer ces différences de performances : ce maïs se comporte en fait comme un maïs physiologiquement moins mûr que son témoin isogène.

Les digestibilités, mesurées en cage sur moutons à l'entretien, ne sont apparemment pas améliorées par le gène waxy.

En conclusion, au niveau fourrager, l'éleveur ne court pas beaucoup de risques en cultivant un maïs waxy pour l'ensilage dans la mesure où il s'assure de la destination fourragère et non céréalière de la récolte.

Mais, utilisé en production laitière, le maïs waxy ne répond pas aux exigences actuelles des transformateurs (rapport TB/TP accru). Néanmoins, au cas par cas, certains éleveurs peuvent être intéressés par un tel fourrage (sous réalisation par rapport au quota, TB particulièrement faible).

\begin{tabular}{lccccc}
\hline & \multicolumn{2}{c}{ essai 1 } & \multicolumn{3}{c}{ essai 2} \\
\cline { 2 - 6 } & témoin isogène & waxy & témoin isogène & témoin dis gène & waxy \\
\cline { 2 - 6 } $\begin{array}{l}\text { Nbre de vaches } \\
\% \text { MS de l'ensilage }\end{array}$ & 19 & 19 & 18 & 18 & 18 \\
$\begin{array}{l}\text { Ingestion totale } \\
\text { (kg de MS } / \mathrm{v} / \mathrm{j})\end{array}$ & 37,1 & 33,3 & 33,7 & 32,7 & 31,9 \\
Lait $(\mathrm{kg} / \mathrm{v} / \mathrm{j})$ & 23,7 & 23,8 & & & \\
TB/TP $(\mathrm{g} / \mathrm{kg})$ & 28,0 & 28,6 & 22,8 & 21,5 & 22,6 \\
& $39,4 / 30,9$ & $40,8 / 31,7$ & $40,2 / 31,3$ & $41,4 / 31,6$ & $41,3 / 31,6$
\end{tabular}

